\title{
Viewing Learning through a New Lens: The Quantum Perspective of Learing
}

\author{
Katherine J. Janzen ${ }^{1}$, Beth Perry ${ }^{2}$, Margaret Edwards ${ }^{2}$ \\ ${ }^{1}$ Faculty of Health and Community Studies, Mount Royal University, Calgary, Canada \\ ${ }^{2}$ Faculty of Health Disciplines, Athabasca University, Athabasca, Canada \\ Email: kjjanzen@mtroyal.ca
}

Received April 11 ${ }^{\text {th }}, 2012$; revised May 12 ${ }^{\text {th }}, 2012$; accepted May $28^{\text {th }}, 2012$

\begin{abstract}
We are living in a quantum world where virtuality allows us to transcend time and space. Boundaries, which were considered to be predetermined, are no longer absolute. This has important implications for the field of education as educators advance e-learning. However, education theory has been outpaced by practice. In this paper the authors propose a new learning perspective - the quantum perspective of learning which moves beyond current popular educational theories of constructivism (Siemens, 2005) and connectivism (Vygotsky, 1978). The five assumptions of the quantum perspective of learning are explored. Specifically, learning is multi-dimensional, occurs in various planes simultaneously, consists of potentialities which exist infinitely, is holistic/holographic in nature and is patterned within holographic realities, and learning environments are living systems. Implications that arise from this perspective are discussed.
\end{abstract}

Keywords: Quantum Perspective of Learning; Pedagogy; Holism; Holographic; Potentialities; Living Systems; Quantum States; Quantum Dimensions; E-Learning

\section{Introduction}

Technology is rapidly developing making boundaries which were considered absolute no longer so. The virtual world has largely transcended time and space. Digital technology allows individuals to virtually co-exist in several places at one time (Andone, Dron, Boyne, \& Pemberton, 2006) often simultaneously interacting with innumerable others with the touch of a screen. We have begun to experience living in a quantum world without limits and constraints. This change has implications for education, as e-learning becomes common.

Developments in educational theory compatible with e-learning have been outpaced by advances in practice. New theory is needed. Emerging learning theories serve two purposes (Kerr, 2007). They replace outdated inferior theories and they address gaps in current theory which older theories do not explain (2007). However, no single model, perspective, or theory exists that explains all learning phenomenon (Calvani, 2008). Hence, a new learning perspective - the quantum perspective of learning (QL) - was conceptualized by the authors to begin to address existing gaps and to establish a common ground for bridging existing learning theories. QL does not explain all learning phenomenon, however, the principles of QL open an effectual door for dialogue.

To situate QL within existing learning theory, six theories are reviewed. The primary principles of quantum mechanics (the foundation of quantum learning), key definitions, and five principle assumptions of QL are described. Implications arising from QL are presented.

\section{Learning Theories: A Review}

Notable learning theories of the last century are behaviouralism (Skinner, 1954), cognitivism (Dewey, 1916; Piaget, 1960/1981), invitational theory (Purkey, 1992), experiential learning (Kolb,
1994), and constructivism (Vygotsky, 1978). Recently, connectivism (Siemens, 2005, 2006) emerged and has gained attention in online education. A brief review of these theories provides a foundation for exploring QL.

\section{Behaviouralism}

Behaviourism regards learning as observable behaviour that results from stimuli, reward, or punishment (Davis, Edmunds \& Kelly-Bateman, 2010). While Locke in the $17^{\text {th }}$ century first identified what is known today as behaviourism, Skinner was the first to officially explicate behaviourism as a learning theory (Williamson, Gunderman, Cohen, \& Frank, 2004). Skinner and other behaviourists that followed believed that it was impossible to determine what went on in the human mind (2004).

To a behaviourist learning results from operant conditioning. Memory occurs when the brain is "hardwired" by repeated experiences. Hardwiring is influenced by reward or punishment where learning is task-based (Davis et al., 2010). A stimulus is repeated until the desired behaviour is observed. Learning is seen as having the product of being right or wrong; correct or incorrect. (Williamson et al., 2004). If a change in behaviour is observed, then learning is thought to have occurred (McLeod, 2003).

\section{Cognitivism}

Cognitivism is attributed to Dewey (1916) and Piaget (1960/ 1981) who were primarily "interested in what went on in the minds of learners" (p. 16). Cognitivists believe that learning is achieved internally rather than externally (McLeod, 2003). The properties of cognitivism are seen as being "structured" or "computational" where prior experiences and current mental patterns (as schema) influence learning (Davis et al., 2010: para. 10). Learning is based on knowledge that is abstract rather than 
concrete (Handley, Sturdy, Finchman, \& Clark, 2006). Memory is a process of encoding, storage, and retrieval and the resultant knowledge is a product of duplication (Davis et al., 2010). The connections of ideas and concepts are important. Knowledge as an end product is "processed on multiple levels," connected to prior experience, and transmitted to new situations (Williamson et al., 2004: p. 16).

\section{Invitational Theory}

Invitational theory and practice (ITP), pioneered by Purkey (1992a) is referred to as "invitational education" where it exists as both a theory of practice (where total learning environments are created) and a theory of learning (Hunter \& Smith, 2007; Paxton, 2003). ITP proposes that effective learning is based on self-concept and that self-concept is vital to successful learning (Riner, 2003).

Humans are considered interdependent, having the utmost authority for their own personal existence, and possessing the ability to "find their own best ways of being and becoming" (Purkey, 1992b: p. 1). Cooperation, empathetic understanding, and genuineness characterize the invitational learning environment (Paxton, 2003). Although ITP has been criticized for the logic of its Pygmalion Effect (self-efficacy), successful learning is viewed as a product of not solely the belief of self and of others, but rather the result of actions stemming from that belief Riner, 2003; Usher \& Pajeres, 2006). In ITP, "living and learning success is nurtured and supported by assisting [learners] in understanding these perceptions and accepting invitations, and opportunities to develop [their] abilities" (Riner, 2003).

\section{Experiential Learning}

Kolb's (1984) experiential learning theory suggests that learning is "the process whereby knowledge is created through the transformation of experience" and posits that learning occurs on a holistic level where the concepts of experience (feeling), perception (perceiving), cognition (thinking), and behaviour (behaving) are integrated into the "total organism" (p. 38).

\section{Constructivism}

The father of constructivism is Vygotsky (1978) with theorists such as Clark (1983, 1985), Papert and Idit (1991), and Lave and Wenger (2002), providing additional insights into this social learning theory. Constructivism is both a learning theory and a philosophy of education (Mattar, 2010). Constructivism as a learning theory proposes that "language" and "scaffolding" exist as the two most important elements in the learning process where social interaction becomes the catalyst for learning (Kop $\&$ Hill, 2008). Through social interaction, a relationship exists between what the individual learner knows and the knowledge that the learner renders from others (2008). Meaning is constructed socially and learning is influenced by the processes of engagement and participation, as well by society and culture (Davis et al., 2010).

\section{Connectivism}

Connectivism has been touted as the "learning theory for the digital age" (Kop \& Hill, 2010: p. 1). Connectivism goes beyond learning within humans to include in learning that is stored and manipulated in forms of technology and organizational learn- ing (Mattar, 2010). Nodes - which can consist of thoughts, feelings, and interactions with others and/or with technology (information sources), and connections which are the links between the nodes, are the basic building blocks of connectivism (Guder, 2010; Siemens, 2005). While constructivism focused on constructing knowledge, connectivism focuses on the constant connections humans make (Siemens, 2006). Learning is seen as processes where "informal information exchange [becomes] organized into networks and [is] supported with electronic tools" (Bessenyei, 2007: p. 11).

\section{Quantum Mechanics: A Foundation for Understanding the Quantum Perspective of Learning}

The primary concepts of quantum mechanics provide background for understanding QL. While quantum mechanics is complex when seen through the eyes of a quantum physicist, the foundational elements which make up quantum mechanics can be readily understood. This understanding begins with the word quantum.

Quantum is derived from the Latin singular quantus meaning "how great" and was first used in quantum mechanics in 1900 by Max Plank (Online Etymology Dictionary, 2011). A quantum is the smallest unit of a physical entity that exists, yet is the most substantial component of quantum mechanics (Princeton University, 2011). This paradox underscores both the complexity and simplicity of quantum mechanics.

Deconstruction in its simplest form allows humans to delineate their assumptions of the world (Longuenesse, 2001). In physics these assumptions diverge into two schools of thought: classical mechanics and quantum mechanics (Bohm, 1973; Raković, 2007). Classical mechanics considers the world to be reducible to machine-like parts which are measurable, orderable, classifiable, and which operate under immutable laws (Bohm, 1971; Kibble \& Berkshire, 2005; Zaman, 2001). Quantum mechanics on the other hand arises from an organismic view where everything is highly connected (Arntz, Chasse \& Vincente, 2006) and operates upon paradoxical laws - laws which operate in time and space and are governed by a unique set of quantum principles. Classical mechanics explains large scale phenomena or dynamics such as the earth's orbit, or the laws that pertain to energy and motion (Kibble \& Berkshire, 2005; Gallego, 2008). However, it does not explain atomistic or sub-atomistic phenomena such as behaviours of electrons and time-reversal symmetry where events go forward and backward in time (Arntz et al. 2006; Kibble \& Berkshire, 2005; Pribram, 2006). It is within this sub-atomistic world where quantum mechanics begins to explain events within this almost imperceptible atomistic biosphere. Quantum mechanics operates on three main concepts: particles and waves, superposition, and entanglement.

\section{Particles and Waves}

The concept of particles and waves is paradoxical. Particles can be waves, and waves can be particles (Aczel, 2002). Particles are electrons. Electrons demonstrate properties of waves or act as indicators of the behaviour of the electrons or particles (Haberken \& Deepak, 2002). Pribram (2006) describes the relationship of waves and particles. "Waves anticipate the future whereas particles remember the past" where particles are the medium and waves "perturb the medium, making a connection 
between two locations in space and time" (p. 42) Wave "energy is [only] potential until it manifests its effects on a substrate" (p. 42). For example, waves in the ocean are only potential until they transform into breakers which demonstrate their energy through the movement of sand on a beach (2006).

\section{Superposition}

Superposition suggests multiple possibilities exist in waves of potential (Artnz et al., 2006). Before the technological age, it was impossible for an individual to exist in two places at the same time and all actions had a cause and effect sequence (Aczel, 2002). The concept of superposition changes this finite view. To illustrate this, if an individual is in a room with two doors, it is impossible to go through both doors at the same time. Quantum mechanics has demonstrated that an "electron, neutron, or even an atom when presented with a barrier with two slits in it, will go through both of them at once" (p. 250). Hence the particle can be in more than one place at one time occupying multiple positions. A single wave function can have 3000 positions simultaneously and yet remains a single wave function (Cafiero \& Adamowicz, 2001). For example, if gelatin and boiling water are placed in a bowl and stirred, each particle of gelatin is superpositioned with the others as the gelatin solidifies. With time and chilling the gelatin solution is transformed into a viscous and eventually solidified medium. When the bowl is perturbed, just as in a wave function, the jiggling of gelatin causes all other parts of the gelatin to move. Thus particles of gelatin are in superposition.

\section{Entanglement}

Plank noted spaces between particles are made up of energy rather than existing in an empty vacuum-like state (Arntz et al., 2006; Haberken \& Deepak, 2002). The universe operates the same way where "all phenomena are caused by energy transfer" (Hrokopos, 2005: p. 90). The concept of entanglement explains this energy transfer. Where the classical mechanics worldview suggests fragmentation and separation, quantum physicists have shown that the universe operates on principles of unity and connectedness (Rossado, 2008). Taking the principle of superposition further, two or more particles existing in superposition become entangled with one other and exist as one system rather than two distinct particles (Aczel, 2002). These particles do not touch, yet are bound up (Arntz et al., 2006). As in gelatin, each particle of gelatin remains distinct and yet is part of each other particle. The spaces between gelatin particles are not empty but are interconnected making a unified whole.

For example, consider interfacing used in tailoring. Seamstresses sew two pieces of fabric together with plain interfacing in between creating a stronger fabric. The interfacing creates a link or a connection with the other pieces of fabric. While the two fabric pieces do not physically touch each other, they are still bound to each other and become inextricably connected. Through the interfacing each piece of fabric becomes part of the other and exists holistically. In a similar way, Aczel notes that systems of particles (two or more particles) can exist and "share some of the properties of the combined states" (Aczel, 2002: p. 25). In quantum mechanics, unlike the two pieces of fabric which are close in proximity, "two particles that [are] miles, or light years, apart may behave in a concerted way: what happens to one happens to the other instantaneously, regardless of the distance between them" (p. 250).

\section{The Quantum Perspective of Learning: Definitions}

Kop and Hill (2008) note that the underpinnings of any perspective begin with clear definitions of terms. QL perspective builds upon existing terms. The terms quantum, quanta, quantum state, quantum dimension, and intelligence as conceptualized and presented in this paper are offered as foundational terminologies proposed by the authors that are unique and original to QL. All other terms are extensions of previous definitions in the literature. A summary of definitions for all terms related to QL are delineated in Table 1.

\section{The Quantum Perspective of Learning Explored}

To understand QL, it is necessary to understand the four basic building blocks of quantum learning: quantum, quanta, quantum dimensions, and quantum states. QL occurs in bits of information. These bits of information borrow their name from quantum mechanics and are known as quantum. A quantum represents the smallest unit of learning that exists.

Table 1.

Key terms used in the quantum perspective of learning.

\begin{tabular}{|c|c|}
\hline Term & Definition \\
\hline Quanta & The smallest unit of learning that exists. \\
\hline Quantum & Multiple units of learning combined or grouped together. \\
\hline Quantum State & $\begin{array}{l}\text { The conditions or states upon which learning takes place } \\
\text { or is experienced. }\end{array}$ \\
\hline Intelligence & The purest form of quanta that exists in a quantum state. \\
\hline Dimension & $\begin{array}{l}\text { "Single observable pattern[s] which when viewed together } \\
\text { become on indivisble whole." (Bohm, 1971: p. 376, 380) }\end{array}$ \\
\hline $\begin{array}{l}\text { Quantum } \\
\text { Dimension }\end{array}$ & $\begin{array}{l}\text { The intersection of cognitive, behavioural, social, cultural, } \\
\text { spiritual and technologoical dimensions which which tog- } \\
\text { ether create a unified whole. }\end{array}$ \\
\hline Entanglement & $\begin{array}{l}\text { The correlation and interacton of multiple entities which } \\
\text { are not dependent on their spatial position/orientation with } \\
\text { each other (CIO Midmarket, 2010). "The linking of these } \\
\text { entities, even though they may be far apart...immediately } \\
\text { causes change in the other [entities]" (Aczel, 2002: p. ix). }\end{array}$ \\
\hline Holism & $\begin{array}{l}\text { The conception and description of reality as whole entites } \\
\text { instead of parts (Jammer, 1988). }\end{array}$ \\
\hline Holographic & $\begin{array}{l}\text { A multi-dimensional thought, feeling or action/interaction } \\
\text { (Guder, 2012; Siemens, 2005). }\end{array}$ \\
\hline $\begin{array}{l}\text { Holographic } \\
\text { Reality }\end{array}$ & $\begin{array}{l}\text { "Multiple explict manifestations of matter... representing } \\
\text { different outcomes of unfolding a single implicate order } \\
\text { that is infinitely connected" (Gallego, 2008: p. 724). }\end{array}$ \\
\hline Plane & $\begin{array}{l}\text { A multi-dimensional surface in which each dimension int- } \\
\text { ersects, interacts, touches or borders all others (Siemens, } \\
\text { 2005; Gallego, 2008). }\end{array}$ \\
\hline Potentiality & $\begin{array}{l}\text { The activation and realization of possibilities which reflect } \\
\text { stability and predictabilty as well as variability (Marquez, } \\
\text { 2006). }\end{array}$ \\
\hline Living System & $\begin{array}{l}\text { "Open, self-organizing systems [having special character- } \\
\text { istics of life and [interacting] with their environment...by } \\
\text { means of information exchanges (Parent, 2000: para. 2). }\end{array}$ \\
\hline
\end{tabular}


These units or bits of information, when combined or grouped together are known as quanta. Quantum, while they can and certainly do exist singularly, manifest themselves primarily as quanta and exist both within and without the human confines. Within humans, the manifestation of quanta occurs in a wide spectrum of learning outcomes. This learning ranges from imperceptible learning, structured learning, serendipitous learning, states of epiphany, and ultimately reaches toward learning that change not only the world of the individual but also the world of the collective human family. Quanta, also reside within technology where they are made manifest within technological advances. For example, quanta exist in computers in the form of bits and bytes. As technology continues to develop, new forms of quanta will be discovered and named. It is important to note that quanta have always existed and are in and of themselves infinite in nature. Quanta reach into the past, reside in the present, but also exist in the future. In this way quanta exist in terms of time and space in perfect symmetry in both the past and the future (Aczel, 2002).

Quantum dimensions draw together the multiple dimensions of the cognitive, social, cultural, spiritual, behavioural, and technological that has been named as well as other dimensions that have yet to be discovered. In classical learning theory these dimensions primarily exist separately and are expressed as individual learning theories. QL is instead expressed in terms of quantum dimensions where together these individual dimensions create a unified whole. If humans are holistic beings, dismissing or discarding any individual dimension of this holism causes the being to cease to exist. The same concept applies to the universe where all dimensions - named or un-named; discovered or undiscovered - are necessary components of the whole. Quantum dimensions, when expressed in terms of learning, become quantum states.

Quantum states are conditions that exist, upon which learning takes place. These states are predicated upon two principles: 1) quantum states are states of being and 2) quantum states represent states of readiness for learning. While Heidegger proposed a state of being-in-the-world, quantum states exist as both temporal (human) and universal states occurring together. This universality is reflected in a state where "the intelligibility of [the quantum state is articulated] in [such] primordial a manner that it gives rise to a potentiality-for-learning which is genuine and... transparent" (Heidegger, 1962: p. 165).

If quantum states represent states of learning readiness, and if is accepted that learning occurs ubiquitously, it follows that learning can occur either imperceptibly, in a state of heightened awareness, or both. This polarity only exists however within the finite human mind as we "learn" every moment of our existence. Neural brain structures constantly communicate and process information in a continual action of re-wiring or re-integrating the existing neural net (Smith, Hood, Stock, Pimm, \& Lemke, 2006). Even when we sleep, this process continues. QL, however, goes beyond the confines of the human body and also occurs outside of our mortal beings. Everything (human and technological) exists and does so in a quantum state or a state of readiness or perpetual quantum state. Likewise, the universe presents in a quantum state. Learning occurs through constant communication (or in other words entanglement) in the medium of time and space. Therefore quantum states occur based on the principle of superposition and exist in all things, in all places, and at all times. Quantum states are holographic in nature.

Rosado offers that we live in a "divided world" which extends infinitely (Rossado, p. 2075). In this divided world, learning and learning theory is not viewed holistically, but rather as "inherently discrete, distanced and disconnected" (p. 2075). QL draws its precepts from quantum mechanics where learning is seen as a holographic entity and where we navigate space and time in terms of holism (Pribram, 2006). QL does not exist "here or there" but rather comes about "here and there" encompassing "everywhere and everywhen" (p. 43).

QL operates on the quantum principle of entanglement where divisible entities act and interact, and a change in one inextricably enacts a change in the other. Where Siemens sees learning as a process of progressively connecting nodes, QL suggests that the various entities that make up the unified whole of quantum learning are already connected. Learning is the process of discovering those connections (Siemens, 2006). As Lipton (2005) noted,

Einstein revealed that we do not live in a [learning] universe with discrete, physical objects separated by dead space. The [learning] universe is one indivisible, dynamic whole in which energy and matter are so deeply entangled it is impossible to consider them as independent elements. (p. 102)

QL purports that learning comes about through infinite interactions that occur as we temporally and virtually exist. Learning is about the realization of those interactions and how they impact our everyday lives. Learning then, "is not [solely] a motion [or an action]... but rather a state of being" (Rossado, 2008: p. 2081). This state of being is referred to as a quantum state or a state of readiness upon which learning is predicated.

While QL "underlies our everyday experience" it also exists as a "domain" of learning that resides outside our immediate, temporal experience in terms of technology and ultimately in the largely uncharted domains of time and space (Pribram, 2006: p. 43). Pribram illustrates the principle of entanglement with the use of a slide projector image. If the lens is taken out of the projector what is viewed is seen as distributed or entangled. With the lens in, individual entities are visible. The lens makes the difference. QL provides a "lens" to view learning in a different way as "we search for the transformations that lead us from one set of observations to a complementary set" (pp. 45-46) which more fully explains learning.

\section{Assumptions of the Quantum Perspective of Learning}

\section{Assumptions of QL}

QL is based upon five assumptions:

1) Learning is multi-dimensional;

2) Learning occurs in various planes simultaneously;

3) Learning consists of potentialities which exist infinitely;

4) Learning is holistic/holographic in nature and is patterned within holographic realities;

5) Learning environments are living systems.

Each assumption is explained in more detail to move quantum learning forward toward theory.

\section{Learning Is Multi-Dimensional}

While other learning theories focus primarily on one dimension of learning, QL recognizes that learning is multi-dimensional an intersection of the cognitive, behavioural, social, cul- 
tural, and technological. Past and present experiences, in which memory has a significant role to play, shape learning in terms of what has been learned (present and past), role modeling (present and past), exposure to technology (past and present), and cultural customs, norms and mores (present and past). Learning expressed as knowledge is "processed on multiple levels" (Williamson et al., 2004: p. 14) or through various lenses through which we experience our worlds. "Learning is not simply about developing one's knowledge and practice, it also involves understanding who we are and in which communities of practice we belong and are accepted (Hanley et al., 2006: p. 644). Learning is continually negotiated "within and across" multiple dimensions (p. 648). Together the dimensions create a unified whole or what is called a quantum dimension.

Vygotsky saw the learning process working from "the outside in" (Glassman, 2001: p. 3). QL recognizes that learning occurs both within and outside individuals as humans interact with and within their temporal and virtual worlds. Siemens (2004) illustrated this in terms of learning or knowledge existing in "non-human appliances" as well as within humans (p. 3). In this way learning is not only consciously created, but it also exists within technology. These temporal-virtual worlds experience constant change as the pace of technology increases exponentially. What is considered cutting-edge pedagogy today may be brought into question for its pragmatism tomorrow. While our humanness creates an anchor to the temporal world, technology creates an anchor to the virtual world. In the virtual world knowledge is "stored and manipulated by technology" (Mattar, 2010: p. 10). While temporal knowledge or quanta exists in technology because of humans, it would be naïve to assume that as human we are the only creators of quanta. Within the confines of what we have discovered however, quantum learners engage with and choose from an abundance of information-both human and technological. This allows the scope of their worlds to increase and learning begins to occur in multi-planar environments.

\section{Learning Occurs in Various Planes Simultaneously}

Siemens (2006b) identifies that "knowledge structures [are not one-dimensional], hierarchical, flat [or] confined belief spaces" (p. 29). Likewise, learning is not restricted to the Cartesian duality of the mind or body, but exists within cognitive, emotional, social (Calvani, 2008; Clark, 1997), spiritual and technological planes. While each of these elements exists singularly, they continually interface with others creating learning that has both depth and breadth. This interface creates a medium for learning where all elements simultaneously co-exist (Andone et al., 2006).

Returning to the example of the seamstress using interfacing, the surfaces of these multiple planes are connected and yet separate; existing as divisible properties and yet as one. With heatactivated or fusible interfacing polymers exist on one side of the interfacing. When activated by heat and pressure the morphology of the polymers is changed to a tack surface which dries upon the removal of the heat source (Dar et al., 2008). Thus the interfacing becomes fused with the fabric. Applying this example to QL, envision an interfacing that has polymers on both sides of the interfacing which connect multiple layers of fabric with multiple pieces of interfacing. In QL these planes are not flat surfaces but rather holograms existing in all directions. Learning occurs and becomes fused and interconnected (in terms of space) on multiple planes.
To take this precept further, it is posited that learning becomes connected by time as well as space. Within this connection there are realms of knowledge that simultaneously exist and communicate within space and time (See Figure 1). The primary realm of knowledge is quantasic knowledge where intelligence exists in its purest form. Atomistic knowledge pertains to the realm of the atom and subatomic, while temporalistic knowledge relates to the earth and our existence/experience upon the earth. Universalistic knowledge moves outward from temporalistic knowledge and encompasses the knowledge of, or pertaining to, the universe. This can be defined both in terms of what we know about the universe and the knowledge that is possessed by the universe. Through science and space travel, universalistic knowledge is becoming more understood. These realms of knowledge, identified at this point in human history, do not preclude that other realms of knowledge exist either in smaller realms or greater realms. At some point these may be named and identified.

Learning is past, present oriented and future oriented. This is consistent with the principles of quantum physics where time exists in perfect symmetry in both the future and the past (Aczel, 2002). From a human perspective our future hopes, expectations and dreams mediate and influence the learning trajectory. From a technological perspective we are transcending boundaries that limit learning. Learning creates "quantum [relationships which] evoke new [realities] that could have not been predicted by breaking down... [the] relational entities [of the various planes or dimensions of quantum learning] into their individual properties" (University of Oregon, n.d., p. 2). When human and technological perspectives merge, potential is infinite.

\section{Learning Consists of Potentialities Which Exist Infinitely}

Human beings only use a fraction of their mental capacity. Infinite potential exists within humans to create, experience, learn, and grow (Stressing, 2011). Looking at learning from "a [temporal] sense, from the first breath at birth to the last moments before death, each individual exists in a plane of never-ending input and output... [where] organizing this information [becomes] the process of learning" (Janzen, 2010: p. 520). While the human mind processes four billion bits of information per second, we only have awareness of 2000 of these (Arntz et al., 2006). Our awareness of these bits of information (quantum)

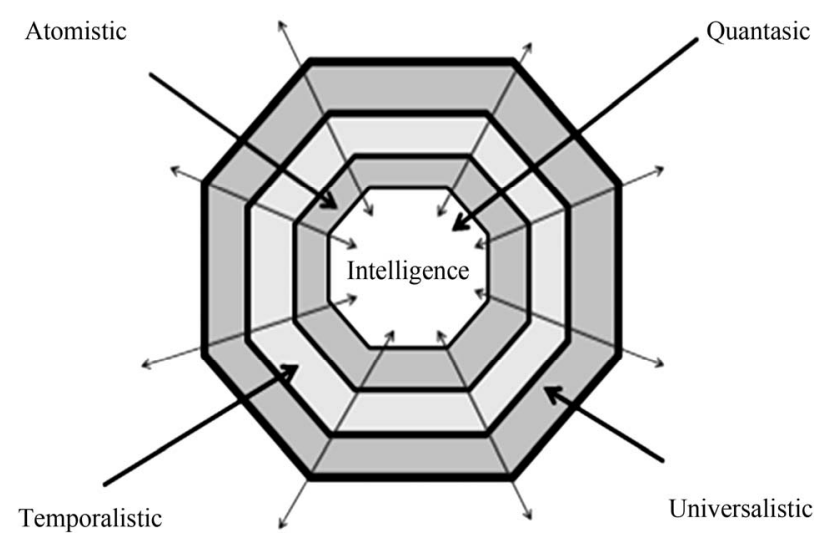

Figure 1.

Realms of knowledge expressed within QL. 
does not negate the presence of the four billion bits of information (quanta) that exist. Likewise our limited awareness of the universe does not negate the existence of an infinite number of quanta. Thus, QL is considered infinite in nature-learning which exists within a time and space without boundaries without beginning or end.

We consider intelligence to be directly correlated with learning potential and cognitive ability. Instead, consider intelligence as a physical entity rather than cognitive ability. Cognitive ability when it is reduced to atomistic proportions is ultimately about chemicals (Pert, 1997). These chemicals, made up of molecules, and are reducible to sub-atomistic elements that communicate and connect via synaptic spaces between neurons in an expansive neural net (Arntz et al., 2006). Learning is purposefully created in these neural nets. This learning, or communication, is not hardwired as the neural net operates upon constant re-rewiring and re-integration (2006). In viewing intelligence as a physical entity there exists infinite potentialities within the body/brain to learn.

Intelligence in $\mathrm{QL}$ is the purest form of quanta that can exist in a quantum state. In the universe and that which is virtual, intelligence is expressed in waves and particles in the form of quantum which entangle and constantly communicate. In the human body intelligence is likewise expressed neurally as neurons connect to all other neurons and communicate in the neural net. Intelligence, when looked at in this way, presents a platform for the existence of infinite potentiality. While we as human beings express this potentiality in many ways, one of the ways intelligence is expressed is the aptitude to construct and develop vision.

Vision allows humans to transcend what it currently known and become future orientated. QL exists within the power of having a vision of what can be, or what can become. The vision of the teacher (whether that be a human teacher or a technological teacher) intersects with the vision of the student. Within the intersection of these two "visions" learning occurs. QL (existing within quantum states) becomes a way of beingin-the-world (Heidegger, 1962) where there are no boundaries except those which we set ourselves (Merleau-Ponty, 1967). In this way learning becomes holographic.

\section{Learning Is Holistic/Holographic and Is Patterned within Holographic Realities}

In a "holographic universe... everything is connected to everything else (Rossado, 2008: p. 2080). Siemens (2004) explains that "people, groups, systems, nodes, entities can [become] connected to create an integrated whole" (p. 3). Likewise it is holographic connections or entanglements that result in both knowledge formation and knowledge translation. Knowledge is not linear (Mattar, 2010) but instead is holographic.

In a temporal sense, finiteness only exists within the learner's perceptions. In a temporal world the capacity to learn grows and expands with time. In the virtual world learning exists infinitely and exponentially. QL allows the virtual world "as [an] invisible, enfolded universe," (Rossado, 2008: p. 2081) to merge with the temporal world. Merging this duality "creates an experience of non-duality" (2085) where the virtual and the temporal are perceived as one and become the learner's reality. Elizabeth Sahtouis, the evolutionary biologist noted

learning and thus intelligence means being able to see the many levels of the whole in space and time and taking them into account when making a decision. It's all about context. The larger your context is the more intelligent your decisions will be. It is about being able to think about different levels of reality at the same time. (Touber, 2006: para. 19)

Holographic realities are about being able to view learning holistically as a continuation of time and space. Holographic realities do not exist on a continuum but rather as holographic matrices that exist in all directions. Consider the presence of waves and particles as quanta existing in superposition and having properties of entanglement. Learning which exists in infinite potentialities occurs within, or is reflected in, the presence of a quantum state. This quantum state is holographic. In a holographic reality learners are not separated from the environment but are a continuation of an environment within a living system. As human beings exist on a sub-atomic level, ultimately we exist in an atomistic reality of waves and particles which interact with other waves and particles. These waves and particles do not differentiate between that which is human and virtual as all exists holistically. Human-virtual environments communicate holistically or as if they were one. It is only our finite minds that see them as separate. QL suggests that learning environments, as part of the holographic reality, also exist as living systems.

\section{Learning Environments Are Living Systems}

QL environments (QLEs) are living systems that become "real" to those who engage with them. Reality becomes a single merged entity, rather than one based on either virtuality or temporality. "Alterations within [QLEs] have ripple effects on the whole" (Siemens, 2004: p. 3) as these environments develop and adapt (Handley et al., 2006).

Consider a pond as a living system. Within the water a multitude of life forms exist in a complex microcosm. A pebble tossed into a pond creates ripples which have an immediate effect on the whole pond. Not only the face of the pond is changed but the pebble may change or affect that which exists within that pond. When learning is introduced into a living system, just as the pebble is introduced into the pond, there are immediate changes in that living system which ripple outward and affect all aspects of that system.

We rarely learn in single units of information (quantum). Rather we learn within multiple bits of information (quanta). To further the analogy of the pebbles and the pond, consider the effect multiple pebbles tossed into the pond at the same time have upon the pond environment. The ripples in the water, which arise from the pebbles, intersect. Even though we only visually see the ripples as being flat, they are instead wave-like and extend everywhere. QLEs are similar. The living learning system as a wavelike system extends in all directions. The difference between the pond and QLEs is that while the pond has finite parameters which we can easily see, quantum learning environments do not. QLEs "are [made up of] connections [where] - everything is connected to everything else" (Touber, 2006: para. 6) and have the ability to grow without the constraints or borders of the pond. QLEs expand, grow and transform through time and space as do the individuals who engage with them.

QLE's unlike the machine-like design of [behavioralist environments] operate according to the principles of living systems. They operate the way nature does - at the edge 
of chaos... They are organic webs of life: dynamic, interconnected networks of relationships that are constantly learning, adapting and evolving (Youngblood, 1997: p. 34).

QLEs take into account humanity and technology. Learning does not take place only within face-to-face or virtual classrooms, quantum learning takes place ubiquitously. With a shift in seeing learning environments as living systems, ultimately the context of learning is enlarged exponentially. Quantum mechanics, when applied to learning theory, allows learning to expand beyond science and technology to become inexplicably connected to humans - humans who are interconnected and interact with the real world (Rossado, 2008).

\section{Overview of Common Assumptions between the Quantum Perspective of Learning and Contemporary Theory}

QL, as mentioned previously, takes elements of contemporary learning theories and builds upon them to create a new perspective. Thus QL neither dismisses nor negates previous theory but instead attempts to come to an understanding about commonalities. It is beyond the scope of this paper to fully explore the intersections of QL and the many forms of learning theory. This will be explored in a future paper. As such, Appendix A presents an overview of the commonalities in assumptions of the six contemporary learning theories that have been presented in this paper and situates them with corresponding assumptions of QL.

\section{Implications and Conclusion}

QL facilitates re-thinking of learning theory by building upon existing theory to explain learning in a fresh and inviting way. QL extracts the best elements of previous learning theory and builds upon them to explain learning in a more holistic sense. The lens of QL, like the lens of a projector, helps us view learning in a clear new light.

Existing learning theories have varying degrees of relevance, being embraced by some and discarded by others. However, we can examine relevant elements of existing theories, and use this analysis to illuminate our understanding of learning. If we negate and refute existing theories without extracting relevant axioms, our efforts to further understand learning could be equated with van Manen's (2002) precept of Writing in the Dark. If we find ourselves "writing in the dark", we are left not only sightless in terms of vision but also directionless in terms of reaching toward the future-for the past influences the future. As the philosopher Merleau-Ponty (1967) so aptly expressed,

I may close my eyes, and stop my ears, I shall nevertheless, not cease to hear, if it is only the blackness before my eyes, or to hear, if only the silence, and in the same way I can "bracket" my opinion or beliefs [or the understanding of learning] that I have acquired, but, whatever I think or decide, it is always against the background of what I have previously believed or done (p. 395).

Our discoveries regarding learning are like realizations encountered when shining a flashlight in the dark (Arntz et al., 2006). Each movement of the flashlight results in new discoveries or paradigms. While the flashlight can only focus on one element at a time, this does not negate the existence of other paradigms or theories that currently exist or that will eventually arise. Imagine a darkened room where one cannot see and visualize the very moment the electrical switch is engaged. What results is a unified paradigm - one which we envision for the very first time. This unified paradigm always existed, but due to our finiteness it is difficult to comprehend without a significant source of illumination. QL helps provide that illumination.

Future learning theories built on what we have known and what we know today are waiting to be explicated and named. Perhaps it is the time to embrace a new paradigm of learning. QL offers an effectual door which we can open and step through in a new view of learning.

\section{Acknowledgements}

This paper was funded by the Social Sciences and Humanities Council of Canada.

\section{REFERENCES}

Andone, D., Dron, J., Boyne, C., \& Pemberton, L. (2006). Are our students digital students? ALT-C 2006: The Next Generation. Conference Proceedings from the 13th International Conference ALT-C, Edinburgh, 5-7 September 2006, 82-96. http://www.alt.ac.uk/altc2006/

Arntz, W., Chasse, B., \& Vincente, M. (2006). What the bleep? Down the rabbit hole. Los Angeles, CA: Lord of the Wind Films.

Bohm, D. (1971). Quantum theory as an indication of a new order in physics. Part A: The development of new orders as shown through the history of physics. Foundations of Physics, 1, 359-384. doi:10.1007/BF00708585

Bohm, D. (1973). Quantum theory as an indication of a new order in physics. B. Implicate and explicate order in physical law. Foundations of Physics, 3, 139-168. doi:10.1007/BF00708436

Cafiero, M., \& Adamowicz, W. (2001). Simultaneous optimalzation of molecular geometry and the wave function in a basis of Singer's n-electron explicitly correlated Gaussians. Chemical Physics Letters, 3355, 404-408. doi:10.1016/S0009-2614(01)00086-0

Calvani, A. (2008). Connectivism: New paradigm or fascinating potpourri? Journal of E-learning and Knowledge Society, 4, 247- 252.

Clark, A. (1997). Being there: Putting brain, body and world together again. Cambridge, MA: MIT Press.

Dar, Y. L., Yuan-Hoffman, W., Xio, A., Shah, S., Huang, D., Hartman, E. et al. (2008). Heat activated pressure sensitive adhesives. http://www.pstc.org/files/ public/Dar08.pdf

Davis, C., Edmunds, E., \& Kelly-Bateman, V. (2010). ConnectivismEmerging perspectives on teaching and technology. http://projects.coe.uga.edu/ epltt/index.php?title=Connectivism

Gallego, M.B. (2008). Physics, consciousness and transcendence: The physics of Roger Penrose and David Bohm as regards for explanation of the human mind open to reality. Pensamiento, 64, 715-739.

Glassman, M. (2001). Dewey \& vygotsky: Society, experience and inquiry. Educational Researcher, 30, 3-14. doi: 10.3102/0013189X030004003

Haberkern, T., \& Deepak, N. (2002). Grains of mystique: Quantum physics for the layman. http://www.opencontent.org/openpub/

Harokpos, E. (2005). Power as the cause of motion and a newfoundation of classical mechanics. Progress in Physics, 2, 80-91.

Heidegger, M. (1962). Being and time. New York, NY: Harper San Francisco.

Hunter, M., \& Smith, K. H. (2007). Inviting school success: Invitational education and the art class. Journal of Invitational Theory and Practice, $13,8-15$.

Janzen, K. J. (2010). Alice through the looking glass: The influence of self and student understanding on role actualization in novice clinical nurse educators. Journal of Continuing Education in Nursing, 41, 517-523. doi:10.3928/00220124-20100701-07 


\section{K. J. JANZEN ET AL.}

Jammer, M. (1988). David Bohm and his work-On the occasion of his seventieth birthday. Foundations of Physics, 18, 691-699. doi:10.1007/BF00734150

Kerr, B. (2007). Msg. 18, Re: What connectivism is. Online Connectivism Conference. Winnipeg: University of Manitoba. URL (last checked 5 February 2007).

http:/ltc.umanitoba.ca/moodle/mod/forum/discuss.php?d=12

Kibble, T. W. B., \& Berkshire, F. H. (2005). Classical mechanics. London: Imperial College Press.

Kop, R., \& Hill, A. (2008). Connectivism: Learning theory of the future or vestige of the past? International Review of Research in Open and Distance Learning, 9, 1-13.

Kolb, D. A. (1984). Experiential leaning: Experience as the source of learning and development. Englewood Cliffs, NJ: Prentice Hall.

Lave, J., \& Wenger, E. (2002). Legitimate peripheral participation in communities of practice. In R. Harrison (Ed.), Supporting lifelong learning: Volume I: Perspectives on learning (pp. 111-126). London: Routledge Falmer.

Longuenesse, B. (2001). Kant's deconstruction of the principle of sufficient reason. The Harvard Review of Philosophy, 9, 67-87.

Marquez, I. (2006). Knowledge of being v. practice of be coming in higher education: Overcoming the dichotomy in the humanities. Arts \& Humanities in Higher Education, 5, 142-161. doi:10.1177/1474022206063651

Mattar, J. A. (2010). Constructivism and connectivism in education technology: Active, situated, authentic, experiential, and anchored learning.

http://www.joaomatar.com/Constructivism\%20and\%20Connectivism $\% 20$ in\%20Education\%20Technology.pdf

Merleau-Ponty, M. (1962). Phenomenology of perception. London: Routledge \& Kegan Paul.

Online Etymology Dictionary (2011). Quantum. http://www.etymonline.com/index.php?search=quantum\&searchmod $\mathrm{e}=$ none

Papert, S., \& Idit, H. (1991). Constructionism. Norwood, NJ: Ablex.

Paxton, P. (2003). Inviting e-learning: How hard can it be? Journal of Invitational Theory and Practice, 9, 23-40.

http://www.invitationaleducation .net/JITP \%20Vol\%208-1.pdf

Pert, C. B. (1997). Molecules of emotion: Why you feel the way you feel. New York, NY: Simon \& Shuster.

Pribram, K. (2006). Holism vs wholism. World Futures, 62, 42-46. doi:10.1080/02604020500406255
Princeton University (2011). Quantum.

http://wordnetweb.princeton.edu/perl/webwn ?s=quantum

Purkey, W. W. (1992a). An introduction to invitational theory. Journal of Invitational Theory and Practice, 1, 5-15.

http://www.invitationaleducation.net/journal/JITP\%20Vol\%208-1.pdf

Purkey, W. W. (1992b). Conflict resolution: An invitational approach. Journal of Invitational Theory and Practice, 1, 43-49. http://invitational education .net/jour nal/v12/p111.htm

Raković, D. (2007). Scientific bases of the quantum holo-graphic paradigm. Proceedings of the International Conference on Measuring Energy Fields, Kamnik, 13-14 October 2007. http:// www.dejanrakovicfund.org/papers/2007-SLOVENIA. pdf

Schmidt, M. (2010). Learning from teaching experience: Dewey's theory and preservice teacher's learning. Journal of Research in $\mathrm{Mu}$ sic Education, 58, 131-146. doi:10.1177/0022429410368723

Siemens, G. (2004). Connectivism: A learning theory for the digital age. http://www.elearnspace. org/Articles/connectivism.htm

Siemens, G. (2005). Connectivism: Learning as network creation. http://www.astd.oeg/LC/2005/1105_siemens.htm

Siemens, G. (2006a). Connectivism: Learning and knowledge today. The International Review of Research in Open and Distance Learning, 9, 1-13.

http://admin.edna.edu.au/dspace/bitstream/2150/34771/1/gs2006_sie mens.pdf

Siemens, G. (2006b). Connectivism: Learning theory or pastime of the self-amused? http://www.Elearnspace.org/Articles/Connectivism_self-amused.htm

Smith, T., Hood, L., Stock, J., Pimm, S., \& Lemke, J. (2006). Pedagogical sessions. Unifying Themes in Complex Systems, 22, 295-346. doi:10.1007/978-3-540-35872-5_8

Usher, E. L., \& Pajares, F. (2006). Inviting confidence in school: Invitations as a critical source of the academic self-efficacy beliefs of entering middle school students. Journal of Invitational Theory and Practice, 12, 7-16.

Van Manen (2002). Writing in the dark: Phenomenological studies in interpretive inquiry. Winnipeg: The Althouse Press.

Vygotsky, L. S. (1978). Mind and society: The development of higher mental processes. Cambridge, MA: Harvard University.

Zaman, L. F. (2001). Postmodern deconstruction of Newtonian science: A physical-to-social transposition of causality. Theory and Science, 2, $1-18$. 


\section{K. J. JANZEN ET AL.}

Appendix A.

Intersecting assumptions of QL and contemporary theory.

\begin{tabular}{|c|c|c|c|c|c|}
\hline \multirow{2}{*}{$\begin{array}{c}\text { Assumptions of } \\
\text { Contemporary Theory }\end{array}$} & \multicolumn{5}{|c|}{ Assumptions of $Q L \longrightarrow$} \\
\hline & Multi-Dimensional & Multiple Planes & Infinite Potential & $\begin{array}{c}\text { Holistic and } \\
\text { Holographic Realties }\end{array}$ & Living Systems \\
\hline Behaviourism & & & & & $\begin{array}{l}\text { Learning dependent on } \\
\text { elements of } \\
\text { environment } \\
\text { (McLeod, 2004) }\end{array}$ \\
\hline Cognitivism & $\begin{array}{l}\text { Ideas and patterns fit } \\
\text { together } \\
\text { (Williamson et al., 2004) }\end{array}$ & $\begin{array}{c}\text { Knowledge processed on } \\
\text { multiple levels; } \\
\text { knowledge interactive } \\
\text { (Williamson et al., 2004) }\end{array}$ & $\begin{array}{c}\text { Freedom of inquiry }= \\
\text { link to future } \\
(\text { Glassman, 2001) }\end{array}$ & $\begin{array}{l}\text { "Continuity of learning" } \\
\text { (Schmidt, 2010: p. 141) }\end{array}$ & \\
\hline Experientialism & & & $\begin{array}{l}\text { Transformation of } \\
\text { experience } \\
(\text { Kolb, 1984) }\end{array}$ & $\begin{array}{l}\text { Holographic adaptations } \\
\text { (Hunter \& Smith, 2007) }\end{array}$ & \\
\hline Invitationalism & & & $\begin{array}{c}\text { "Realization of } \\
\text { [unlimited] human } \\
\text { potential" } \\
\text { (Purkey, 1992b: p. 2) }\end{array}$ & & \\
\hline Constructivism & $\begin{array}{l}\text { Learning is contextual } \\
\text { (Kaplan, n.d.) }\end{array}$ & & & $\begin{array}{l}\text { Active engagement with } \\
\text { world } \\
\text { (Kaplan, n.d.) }\end{array}$ & $\begin{array}{l}\text { Learning active process } \\
\text { (Kaplan, n.d.) }\end{array}$ \\
\hline Connectivism & $\begin{array}{l}\text { Exchange of information } \\
\text { organized into networks } \\
\text { (Bessenyei, 2007) }\end{array}$ & $\begin{array}{l}\text { "Connections between } \\
\text { fields, ideas and } \\
\text { concepts" } \\
\text { (Guider, 2010: p. 37) }\end{array}$ & $\begin{array}{c}\text { Capacity to know } \\
\text { supersedes current } \\
\text { knowledge (Siemens, } \\
\text { 2004) }\end{array}$ & $\begin{array}{l}\text { Constant connections } \\
\text { (Siemens, 2006) }\end{array}$ & $\begin{array}{l}\text { "Alterations within } \\
\text { network have ripple effect } \\
\text { on whole" } \\
\text { (Siemens, 2004: p. } 3 \text { ) }\end{array}$ \\
\hline
\end{tabular}

